\title{
Lipopolysaccharides Increase the Risk of Colorectal Cancer Recurrence and Metastasis Due to the Induction of Neutrophil Extracellular Traps After Curative Resection
}

\author{
Wei-wei Wang \\ Tinghu People's Hospital \\ Lipei Wu \\ Dongftai People's Hospital \\ Wenying Lu \\ Tinghu People's Hospital

\section{Wei Chen} \\ Tinghu People's Hospital

\section{Wenhui Yan} \\ Tinghu People's Hospital \\ Chunrun Qi \\ Tinghu People's Hospital

\section{Shihai Xuan} \\ Dongtai People's Hospital
}

Anquan Shang ( $\nabla$ shanganquan@tongji.edu.cn )

Shanghai Tongji Hospital https://orcid.org/0000-0003-2820-1982

\section{Research Article}

Keywords: Lipopolysaccharides, Neutrophil Extracellular Traps, Recurrence, Metastasis, Colorectal cancer

Posted Date: May 5th, 2021

DOl: https://doi.org/10.21203/rs.3.rs-474607/v1

License: (9) This work is licensed under a Creative Commons Attribution 4.0 International License. Read Full License 


\section{Abstract}

Background: Intra-abdominal infection after curative surgery for colorectal cancer is a serious complication associated with an increased risk of recurrence. Lipopolysaccharides (LPS) - an essential component of the cell wall of Gram-negative bacteria - were found to exert a protumorigenic effect by stimulating the inflammatory pathology and formation of neutrophil extracellular traps (NETs). This study was conducted to test whether LPS-induced formation of NETs promotes the development of cancer and metastasis.

Methods: The clinical characteristics, incidence of relapse, and serum myeloperoxidase-DNA complexes of 40 patients with infection and 40 patients without infection after curative surgery were analyzed. The effects of LPS on the induction of NETs were evaluated in a mouse model of colorectal cancer and liver metastasis. The toll-like receptor 9 (TLR9) - a DNA receptor - was knocked down to assess its effect on the mitogen-activated protein kinase pathway and activities implicated in the formation of NETs.

Results: Analysis of the clinical data obtained from these patients showed the significant relation of the formation of NETs and incidence of metastasis and survival rates. Subsequent in-vitro experiments revealed an increased level of citrullinated-histone H3 and myeloperoxidase-DNA in LPS-injected mice with colorectal cancer. In the mimic metastatic model, injection of LPS enhanced the metastatic capacity, which was then attenuated by DNase I. This suggested that the formation of NETs was activated by LPS. Injection of TLR9-knockdown HCT116 cells in mice, followed by induction through LPS, mitigated the level of citrullinated-histone $\mathrm{H} 3$ and myeloperoxidase-DNA. This finding implied that the formation of NETs was suppressed.

Conclusion: These findings shed light on the mechanism underlying the relationship between the elevated rate of colorectal cancer recurrence in patients who underwent surgery and the incidence of infection. This mechanism involves the protumorigenic activities of LPS-induced formation of NETs. The NETs which could be mediated by the TLR9 and the mitogen-activated protein kinase signaling pathway.

\section{Introduction}

Although resection of the primary tumors is the treatment of choice for patients suffering from colorectal cancer, the risk of recurrence after surgery remains a challenge. Tobe more precise, postoperative septic complications, such as surgical site infections or remote infections, may influence the long-term prognosis after curative surgery for colorectal cancer(McArdle et al. 2005; Mynster et al. 2000; Nespoli et al. 2004; Nowacki and Szymendera 1983). Previous hypotheses assumed that viable tumor cells were present at the anastomotic site, leading to the implantation of tumor cells(van den Tol et al. 1998). In this case, local recurrence may occur due to anastomotic leakage or perineal wound infection(Bell et al. 2003; McArdle et al. 2005; Walker et al. 2004). Additionally, the release of proinflammatory cytokines and growth factors produced by an inflammatory response mechanism may confer a favorable microenvironment to the residual tumor cells(McArdle et al. 2005). Apart from local recurrence, a 
predisposition to metastasis (e.g., liver and lung) following curative surgery has attracted considerable attention(Richards et al. 2011).

Neutrophils serve as the first line of defense of defense against invading infections and pathogens. Despite the fact that the polarization of neutrophils towards a protumorigenic phenotype may be mediated by chemokines released in the tumor microenvironment, it still poses an threat to the recurrence and metastasis of cancer (Sagiv et al. 2015). Neutrophil extracellular traps (NETs) are formed from loose DNA molecules connected to one another in a web-like structure, and are composed of histones, myeloperoxidase (MPO), neutrophil elastase, cathepsin G, and lactoferrin(Urban et al. 2009). These have been shown to promote the progression of cancer and metastasis(Cools-Lartigue et al. 2013). In addition, A previous study showed that lipopolysaccharides (LPS) - a major component of Gram-negative bacteria - may induce the recruitmentof neutrophils and increase the invasive ability of pancreatic cancer cells(Ikebe et al. 2009). Based on these findings, we hypothesized that the formation of NETs was induced by LPS during infection or sepsis, promoting the development of cancer and metastasis.

\section{Materials And Methods}

\section{Ethical statement}

This study was performed under the approval of the Animal Ethics Committee of Tinghu People's Hospital. Extensive efforts were made to ensure animal suffering was minimized.

\section{Patient samples and data}

This study included patients who were disease-free at the end of the operation, with a complete medical record (i.e., tumor location, hospitalization, lymphatic invasion, vascular invasion, intra-abdominal infection, etc.). Serum samples were collected on postoperative day 1 from patients who underwent curative surgery for colorectal cancer at Tinghu People's Hospital, between 2016 and 2020. Disease-free survival was defined as time from the completion of surgery to cancer recurrence, or death from any cause.. Patients who were lost to follow-up had their data censored at the last date ofcontact. Written informed consent was obtained by all patients prior to inclusion, and all materials were obtained following approval by the institutional review board of Tinghu People's Hospital.

\section{Cell culture}

The human colorectal cell line HCT116 was purchased from Sigma-Aldrich (St. Louis, MO, USA). The cells were maintained in a subconfluent state using McCoy's 5a minimum essential media (ThermoFisher, Shanghai, China) supplemented with $10 \%$ fetal bovine serum and $1 \%$ penicillin and streptomycin.

\section{Western blotting}

Cells were lysed in NP-40 buffer containing 150 mM sodium chloride, 1.0\% NP-40, 50 mM Tris hydrochloride, complete ${ }^{\mathrm{TM}}$ Protease Inhibitor Cocktail (Roche Applied Science, Mannheim, Germany), and 
$1 \mathrm{mM}$ phenylmethyl sulfonyl fluoride for 30 minutes on ice. The supernatant was subjected to protein denaturation with buffer containing $2 \%$ sodium dodecyl sulfate (Sigma-Aldrich, St. Louis, MO, USA). Subsequently, it was analyzed using sodium dodecyl sulfate-polyacrylamide gel electrophoresis and a monoclonal antibody against toll-like receptor 9 (TLR9), p38, p-p38, p65, p-p65, c-jun amino-terminal kinase (JNK), p-JNK, Stat, and p-Stat. The any unbound primary antibody was washed away after incubation, and then the membrane incubated with the appropriate horseradish peroxidase-conjugated immunoglobulin secondary antibodies. Afterwards, enhanced chemiluminescence (GE Healthcare) was used to detect the staining.

\section{Neutrophil isolation}

Mouse neutrophils were isolated from bone marrow extracted from the tibias and femurs. Neutrophils were sorted after incubation with allophycocyanin (APC) -conjugated anti-mouse-Ly6G antibody (BD Pharmingen ${ }^{\mathrm{TM}}$, Cat NO. 560599) and APC-Cy7 CD 11b (BD Pharmingen ${ }^{\mathrm{TM}}$, Cat NO. 557657).

\section{Experimental metastasis assay}

Colorectal metastases were induced in mice as previously described(Nicoud et al. 2007). To understand the mechanism of metastasis, the experiment was conducted. Specifically, $1 \times 10^{4} \mathrm{HCT} 116$ cells or luciferase-labeled HCT116 cells (HCT116-GFP-Luc) were injected via a 1-cm midline laparotomy into the spleens of 8- to 10-week-old nude mice. The tumor cells were allowed to circulate for 15 minutes. In the control group. experimental groups respectively. Prior to the injection of LPS, splenectomy was performed to prevent the formation of splenic tumors. Approval of this experimental protocol was obtained from the Committee on the Ethics of Animal Experiments of Xi'an Jiaotong University (Approval Number: 2011045).

\section{Detection and quantification of MPO-DNA and citrullinated-histone H3 (Cit-H3)}

The detection of serum MPO-DNA complexes was performed using the enzyme-linked immunosorbent assay, as previously described (Nakazawa et al. 2014). In brief, microtiter plates (Thermo Fisher Scientific, Waltham, MA, USA) were initially coated with the monoclonal anti-human MPO antibody $(5 \mu \mathrm{g} / \mathrm{ml}$; AbD Serotec, Kidlington, UK) and incubated overnight at $4^{\circ} \mathrm{C}$. After blocking with $1 \%$ bovine serum albumin, the sera (final dilution 1:3) and horseradish peroxidase-conjugated anti-DNA antibody in the cell death detection kit (Roche Diagnostics, Tokyo, Japan) were added to the wells, then peroxidase substrate was added according to the instructions provided by the manufacturer. The serum from a patient with MPOantineutrophil cytoplasmic antibody-associated vasculitis served as a positive control.

Colon sections were prepared and mounted on glass slides to detect $\mathrm{Cit}-\mathrm{H} 3$ in tissues. Antigen retrieval was performed using citrate buffer and then the retrieved specimens were permeabilized with $0.1 \%$ Triton $\mathrm{X}-100$ for $10 \mathrm{~min}$. Subsequently, specimens were blocked with phosphate-buffered saline containing 3\% bovine serum albumin and $1 \%$ donkey serum. The sections were incubated with primary antibodies, specifically anti-citrullinated-histone H3 (1:100; Abcam, Cambridge, MA, USA). This was followed by 
incubation with Alexa Fluor 488 donkey anti-rabbit (1:500; Abcam) and Alexa Fluor 647 donkey anti-goat (1:500; Abcam) secondary antibodies overnight at $4^{\circ} \mathrm{C}$, respectively. For the detection of DNA, 4'6diamidino-2-phenylindole was applied.

\section{Treatment with LPS, DNase I, and YW4-03}

Initially, injections of LPS (5 $\mu \mathrm{g} / \mathrm{ml})$, DNase I (50 mg/mouse, Roche), or YW4-03 (10 mg/kg) were administered immediately prior to abdominal closure. This was followed by daily administration of intraperitoneal doses of DNAse (thrice weekly for YW4-03).

\section{Statistical analysis}

For the animal studies, the results are expressed either as the mean \pm standard error of the mean or the mean \pm standard deviation. One-way analysis of variance was used for group comparisons, whereas Student's t-test was applied in Comparisons between the experimental and control groups. For the data analysis involving human subjects, human patients, we dichotomized the levels of the MPO-DNA complex at the median and compared the baseline characteristics for each group. Besides categorical variables were examined using the chi-squared test or Fisher exact tests, while continuous variables were analyzed using Student's t-test or the Wilcoxon rank-sum test. The analysis of disease-free survival was conducted using the Kaplan-Meier method. For all analyses, a two-tailed $p<0.05$ denoted statistical significance.

\section{Results}

\section{Relapse and metastasis are associated with the widespread formation of NETs}

Multiple lines of evidence showed that LPS may induce the formation of NETs. In experimental models and patients with cancer, deposition of NETs displayed a potential association with tumor progression in tumor tissues(Berger-Achituv et al. 2013; Cools-Lartigue et al. 2013; Sangaletti et al. 2014). We hypothesized that the LPS produced during postoperative infection may also induce the formation of NETs, contributing to the elevated rate of relapse. To examine this hypothesis, we recruited 120 patients diagnosed with non-metastatic colorectal cancer. Forty patients with anastomotic leakage or intraabdominal abscess were classified in the infection group. Forty other patients without postoperative complications were included in the control group. In addition, 40 healthy volunteers were included in this study as healthy controls. (Table 1). In the infection group, 23 patients required reoperation. Moreover, the average length of hospitalization was longer versus that reported in the control group.

Except neutrophils, the number of other components in immune cells was similar particularly.. Furthermore, the Kaplan-Meier analysis evaluated the association between recurrence and infection status. The results showed that the rate of 2-year cumulative recurrence was significantly higher in the infection group versus the control group (Fig. 1A, $p=0.00128$, Wilcoxon log-rank test). 
MPO-DNA complexes were measured to indicate the nucleosomes derived from NETs, thereby quantifying the degree of NETs formation(Sangaletti et al. 2014; Yoo et al. 2014). There was a significant increase in the formation of NETs in the infection group, compared with that reported in patients without complicated inflammation (Fig. 1B). In the analysis of disease-free survival, the patients were grouped into low MPO-DNA $(n=18)$ and high MPO-DNA $(n=22)$ groups according to the comparison with the median level of optical density (OD) of MPO-DNA (OD = 0.323). Two patients in the high MPO-DNA group expired during the follow-up period. The risk of recurrence in the high MPO-DNA group was higher than that reported in the low MPO-DNA group (Fig. 1C, $p=0.0209$, chi-square test). The association between clinical parameters, including the level of MPO-DNA, infection status, and recurrence is presented in Table 2. The bivariate analysis showed that the level of MPO-DNA in the serum, intraabdominal infection, the concentration of interleukin 6 in the peritoneal fluid, vascular invasion, and perineural invasion were significantly associated with recurrence.

\section{LPS promotes the formation of NETs in a mouse model of colorectal cancer}

We injected LPS into the peritoneal cavity of mice with colorectal cancer and performed immunofluorescence staining of colon sections to investigate the role of LPS in promoting the formation of NETs in colorectal cancer. Phosphate-buffered saline was administered to the control group. The formation of NETs - identified based on the co-localization of extracellular fibrillary and web-like structures with Cit-H3 - was observed in the blood of experimental mice (Fig. 2A, 2B, and 2C). Following pretreatment with DNase I, a small amount of MPO-DNA was also detected in the serum, suggesting that the formation of NETs was reduced (Fig. 2D). In the colon tissue of control mice, there was no formation of NETs observed.

\section{LPS promotes metastasis, which is attenuated by treatment with DNase I}

We subsequently sought to determine whether the LPS-induced formation of NETs is associated with the development of metastasis. Tumor-cell shedding into the circulation is a primary potential origin of metastasis after surgery(Weitz et al. 2000). Therefore, prior to treatment with LPS, we mimicked metastasis by injecting HCT116-GFP-Luc into the spleen of mice and allowing the cells to circulate for 15 min. Following the establishment of a metastatic model, intraperitoneal administration of LPS was performed. The level of Cit-H3 in liver tissues sectioned from LPS-treated mice was significantly higher than that observed in liver tissues of control mice (Fig. 3A and 3B). Similarly, we detected a significantly reduced concentration of circulating MPO-DNA complexes in the serum of DNase I-treated mice (Fig. 3C). Bioluminescent imaging showed that mice treated with DNase I after injection of LPS exhibited significantly reduced metastasis versus that reported in mice treated only with LPS (Fig. 3D).

We subsequently evaluated the effect of NETs suppression using a different strategy. Peptidyl arginine deiminase type IV is essential for the citrullination of histone-H3. This is a critical step during the formation of NETs, as are chromatin decondensation and expulsion(Hemmers et al. 2011). We used a peptidyl arginine deiminase type IV inhibitor - YW4-03 - to treat the mice after injection of HCT116 cells 
into the spleen. A significant decrease in metastatic capacity was observed, and the level of Cit-H3 was substantially reduced (Fig. 3E).

\section{TLR9 is involved in the mitogen-activated protein kinase (MAPK) pathway}

The MAPK pathway is a canonical protumorigenic pathway. A previous study showed that LPS may promote the MAPK pathway(Anton et al. 2012). Considering that both NETs and the MAPK pathway are enhanced by LPS, we hypothesize that the formation of NETs may be related to the MAPK pathway. TLRs have been reported as mediators of cell activation via pathways, including MAPK and nuclear factor kappa-B (NF-kB), resulting in substantial elevation in the transcription of cytokine and chemokine genes(So and Ouchi 2010). Furthermore, TLR9 has been reported as a cellular DNA receptor implicated in activities related to cancer pathology (Gao et al. 2013; Moreira et al. 2015). Therefore, we hypothesized that TLRs may play an important role in tumor progression enhanced by the formation of NETs. In the colorectal mouse model, we found that TLR9, p-p38, p-p65, p-JNK, and p-Stat were significantly increased after the injection of LPS. In contrast, treatment with DNase I significantly decreased their expression (Fig. 4A). In vitro, the HCT116 cell line was cultured using media derived from LPS-stimulated neutrophils. The expression of TLR9 was significantly increased compared with that determined in HCT116 cells cultured using control media. The proteins implicated in the activation and downstream of MAPK pathway, p-p38, p-p65, p-JNK, and p-Stat, were also increased in HCT116 cells cultured in conditioned media (Fig. 4B).

We subsequently knocked down TLR9 in HCT116 cells and cultured those TLR9-deficient cells in media derived from LPS-induced neutrophils. Western blotting showed that the levels of the proteins TLR9, pp38, p-p65, p-JNK, and p-Stat were significantly reduced (Fig. 4C). In parallel, tumor cell proliferation, migration, and invasion were substantially decreased (Fig. 4D).

\section{Discussion}

Colorectal cancer is one of the most common malignancies, ranking third among cancers in terms of mortality. Surgery is an effective treatment option for colorectal cancer. However, the occurrence of intraabdominal infection after surgery is a serious complication, with a reported incidence ranging from $0.5 \%$ to $>30 \%$ (Bruce et al. 2001; Chambers and Mortensen 2004; Serra-Aracil et al. 2011). Notably, in some series, this incidence reached an alarming 40\%(Bohle et al. 2010; Cong et al. 2013; Katoh et al. 2011; Mirnezami et al. 2011). reported that the development of a postoperative complication due to intraabdominal infection is associated with a higher risk of morbidity and mortality(Bohle et al. 2010), and often related to liver metastasis. The present study revealed a significant association between the occurrence of postoperative infection and an elevated rate of relapse. The mechanism underlying this association can be demonstrated via the correlation between the formation of NETs and infection events.

Emerging evidence highlights neutrophils as the first immune barrier during infections, and therefore promoting the development of tumors. NETs are web-like structures composed of extruded DNA and antimicrobial proteins(Brinkmann et al. 2004; Clark et al. 2007; Fuchs et al. 2007; Metzler et al. 2011; 
Pilsczek et al. 2010). Studies using sepsis models have shown that NETs trap circulating pathogens within the liver(McDonald et al. 2009). However, NETs are also characterized by their protumorigenic roles. Notably, excessive formation of NETs may enhance tumor-cell shedding into the circulation through surgery or detachment from the tissue, facilitating metastasis. Spicer et al. demonstrated that circulating tumor cells - like microbial cells - may be captured by NETs in postoperative sepsis, thereby promoting the adhesion and dwelling of tumor cells in tissues of distant organs(Cools-Lartigue et al. 2013). Moreover, the high concentration of proteins released during the formation of NETs may induce proliferation and inhibit apoptosis of tumor cells. Considering the significant association between infection and disease recurrence, we hypothesized that NETs may be the link connecting infection and metastasis in colorectal cancer. In the present study, a significant increase in the formation of NETs was detected in the infection group. The risk of recurrence in the high MPO-DNA group was higher than that reported in the low MPO-DNA group. The bivariate analysis showed that the level of MPO-DNA in the serum, intra-abdominal infection, the concentration of interleukin 6 in the peritoneal fluid, vascular invasion, and perineural invasion were significantly associated with recurrence. Collectively, we can establish the link between the formation of NETs and development of tumor.

Evidence suggests that systemic inflammation and postoperative infection result in cancer recurrence(McMillan et al. 2003; Miki et al. 2008; Nespoli et al. 2004). However, the mechanisms underlying the higher risk of cancer recurrence in the presence of Gram-negative bacterial infections remain elusive. LPS an essential component of the outer membrane of Gram-negative bacteria, has been shown to increase the recruitment of cancer cells to hepatic sinusoids(McDonald et al. 2009) and liver metastasis (Vidal-Vanaclocha et al. 1996) by inducing systemic inflammation. Rich et al. demonstrated that LPS-induced TLR4 signaling in human colorectal cancer cells increases $\beta 1$ integrin-mediated cell adhesion and liver metastasis(Hsu et al. 2011). In patients with sepsis, platelets can be activated by TLR4, and subsequently combined with neutrophils to produce NETs, leading to tissue damage and dysfunction of coagulation(Clark et al. 2007). Jie et al. demonstrated that TLR4 signaling may augment the migration of chemokine-induced neutrophils by modulating the expression of chemokine receptors on the cell surface(Fan and Malik 2003). These findings support the postulation that the LPS-induced formation of NETs may promote the development of cancer. The present study demonstrated that the formation of NETs in the colon tissue of experimental mice after treatment with LPS was attenuated by treatment with DNase I. In a mimic metastatic model, the LPS-treated mice exhibited extensive migration of tumor cells and widespread formation of NETs, which was reduced by treatment with DNase I. This finding was confirmed using another NETs inhibitor (YW4-03).

The MAPK signaling pathway is an evolutionarily conserved transduction pathway mediating inflammatory processes(De Nardo et al. 2009; Kogut et al. 2005; Yang et al. 2011). The extracellular signal-regulated protein kinase 1/2 (ERK), p38, and JNK are three major groups of the MAPK. Abdul et al. discovered that NETs were induced by treatment with phorbol-12-myristate-13-acetate(Brinkmann et al. 2004; Fuchs et al. 2007). Consequently, they identified specific proteins that implicate the Raf-MEK-ERK pathway in the formation of NETs. In the Raf-MEK-ERK pathway, Ras activates Raf, which initiates MEK1/2 signaling and downstream Erk1/2, thereby prmoting cell proliferation and survival. Consistently, 
the present study revealed a significant increase in TLR9, p-p38, p-p65, p-JNK, and p-Stat - major components of the MAPK pathway - in a mouse model of colorectal cancer after injection of LPS. This effect was reduced through treatment with DNase I. In vitro, the expression of TLR9 was determined in HCT116 cells cultured using media derived from LPS-stimulated neutrophils. TLRs - the upstream activator of the MAPK pathway - are phylogenetically conserved transmembrane proteins involved in the induction of innate and adaptive immune responses and detection of pathogens(Peroval et al. 2013). Antigens derived from pathogens may stimulate TLRs to produce proinflammatory mediators, such as cytokines and nitric oxide. TLR9 plays a critical role in the LPS-induced formation of NETs. As a protein of the TLR family, TLR9 showed a particular function related to DNA(Füri et al. 2013; Zannetti et al. 2014) and was thus considered a candidate effector. In the present study, HCT116 cells cultured in the media derived from LPS-induced neutrophils expressed elevated levels of proteins implicated in the MAPK signaling pathway (i.e., TLR9, p-p38, p-p65, p-JNK, and p-Stat). Notably, TLR9-deficient HCT116 cells cultured in media derived from LPS-induced neutrophils exhibited reduced expression of these proteins. Accordingly, tumor cell proliferation, migration, and invasion were substantially decreased.

\section{Conclusion}

The findings of the present study shed light on the mechanism underlying the relationship between the elevated rate of colorectal cancer recurrence in patients who underwent surgery and the incidence of infection. The results implicate the LPS-induced formation of NETs in promoting the development of tumors and metastasis. The TLR9 was found to be responsible for the formation of NETs in LPS-induced neutrophils, and MAPK pathway is activated in the course of NETs formation. Further studies are warranted to address the suitability of TLR9 as a target for the reduction of disease recurrence in patients with infections.

\section{Abbreviations}

ELISA enzyme-linked immuno sorbent assay

LPS Lipopolysaccharides

NETs neutrophil extracellular traps

NE neutrophil elastase

Cit-H3 citrullinated-histone H3

MPO myeloperoxidase

TLR9 toll-like receptor 9

CRC Colorectal cancer 


\section{Declarations}

\section{Acknowledgments}

The authors are thankful to the doctors and nurses of Tinghu Hospital and Dongtai Hospital, and Shanghai Dobai Biomedicin Technology CO., LTD for their keen support of this research.

\section{Author contributions}

AQS conceptualized the whole research and guided the direction and progress of it. WWW and CRQ performed the biological experiments, WC and WHY interpreted the results and data, WWW drafted the manuscript. LPW, WYL and SHX recruited patients.

\section{Funding}

This work was supported by the following: The Medical Research Project of Jiangsu Provincial Health and Health Commission (2019179); Shanghai Post-doctoral Excellence Program (2020409), The Postdoctoral Science Foundation of China (2020M681399); The 2021 National Natural Science Foundation of Shanghai Tongji Hospital Incubation Project (TJ202010).

\section{Data availability}

The datasets supporting the conclusions of this article are included within the article and its additional file

\section{Conflict of interest}

The authors declare that they have no conflict of interest.

\section{Ethics approval}

The study was approved by the ethics committee of the Fudan University Shanghai Cancer Center and signed informed consent was obtained from each patient. All animal procedures were performe according to national guidelines and approved by the Institutional Com-mittee of Fudan University for Animal Research.

\section{Consent to participate}

Informed consent was obtained from all par-ticipants included in this study.

\section{Consent for publication}

All authors have consented to publication of the results presented in this manuscript.

\section{References}


1. Anton L, Brown AG, Parry S, Elovitz MA (2012) Lipopolysaccharide induces cytokine production and decreases extravillous trophoblast invasion through a mitogen-activated protein kinase-mediated pathway: possible mechanisms of first trimester placental dysfunction. Hum Reprod 27(1):61-72. https://doi.org/10.1093/humrep/der362

2. Bell SW, Walker KG, Rickard MJ, Sinclair G, Dent OF, Chapuis PH et al (2003) Anastomotic leakage after curative anterior resection results in a higher prevalence of local recurrence. $\mathrm{Br} J$ Surg 90(10):1261-1266. https://doi.org/10.1002/bjs.4219

3. Berger-Achituv S, Brinkmann V, Abed UA, Kühn LI, Ben-Ezra J, Elhasid R et al (2013) A proposed role for neutrophil extracellular traps in cancer immunoediting. Front Immunol 4):48. https://doi.org/10.3389/fimmu.2013.00048

4. Bohle B, Pera M, Pascual M, Alonso S, Mayol X, Salvado M et al (2010) Postoperative intraabdominal infection increases angiogenesis and tumor recurrence after surgical excision of colon cancer in mice. Surgery 147(1):120-126. https://doi.org/10.1016/j.surg.2009.06.035

5. Brinkmann V, Reichard U, Goosmann C, Fauler B, Uhlemann Y, Weiss DS et al (2004) Neutrophil extracellular traps kill bacteria. Science 303(5663):1532-1535. https://doi.org/10.1126/science.1092385

6. Bruce J, Krukowski ZH, Al-Khairy G, Russell EM, Park KG (2001) Systematic review of the definition and measurement of anastomotic leak after gastrointestinal surgery. Br J Surg 88(9):1157-1168. https://doi.org/10.1046/j.0007-1323.2001.01829.x

7. Chambers WM, Mortensen NJ (2004) Postoperative leakage and abscess formation after colorectal surgery. Best Pract Res Clin Gastroenterol 18(5):865-880. https://doi.org/10.1016/j.bpg.2004.06.026

8. Clark SR, Ma AC, Tavener SA, McDonald B, Goodarzi Z, Kelly MM et al (2007) Platelet TLR4 activates neutrophil extracellular traps to ensnare bacteria in septic blood. Nat Med 13(4):463-469. https://doi.org/10.1038/nm1565

9. Cong ZJ, Hu LH, Bian ZQ, Ye GY, Yu MH, Gao YH et al (2013) Systematic review of anastomotic leakage rate according to an international grading system following anterior resection for rectal cancer. PLoS One 8(9):e75519. https://doi.org/10.1371/journal.pone.0075519

10. Cools-Lartigue J, Spicer J, McDonald B, Gowing S, Chow S, Giannias B et al (2013) Neutrophil extracellular traps sequester circulating tumor cells and promote metastasis. J Clin Invest . https://doi.org/10.1172/JCl67484

11. De Nardo D, De Nardo CM, Nguyen T, Hamilton JA, Scholz GM (2009) Signaling crosstalk during sequential TLR4 and TLR9 activation amplifies the inflammatory response of mouse macrophages. J Immunol 183(12):8110-8118. https://doi.org/10.4049/jimmunol.0901031

12. Fan J, Malik AB (2003) Toll-like receptor-4 (TLR4) signaling augments chemokine-induced neutrophil migration by modulating cell surface expression of chemokine receptors. Nat Med 9(3):315-321. https://doi.org/10.1038/nm832

13. Fuchs TA, Abed U, Goosmann C, Hurwitz R, Schulze I, Wahn V et al (2007) Novel cell death program leads to neutrophil extracellular traps. J Cell Biol 176(2):231-241. 
https://doi.org/10.1083/jcb.200606027

14. Füri I, Sipos F, Spisák S, Kiszner G, Wichmann B, Schöller A et al (2013) Association of self-DNA mediated TLR9-related gene, DNA methyltransferase, and cytokeratin protein expression alterations in HT29-cells to DNA fragment length and methylation status. ScientificWorldJournal 2013):293296. https://doi.org/10.1155/2013/293296

15. Gao C, Kozlowska A, Nechaev S, Li H, Zhang Q, Hossain DM et al (2013) TLR9 signaling in the tumor microenvironment initiates cancer recurrence after radiotherapy. Cancer Res 73(24):7211-7221. https://doi.org/10.1158/0008-5472.CAN-13-1314

16. Hemmers S, Teijaro JR, Arandjelovic S, Mowen KA (2011) PAD4-mediated neutrophil extracellular trap formation is not required for immunity against influenza infection. PLoS One 6(7):e22043. https://doi.org/10.1371/journal.pone.0022043

17. Hsu RY, Chan CH, Spicer JD, Rousseau MC, Giannias B, Rousseau S et al (2011) LPS-induced TLR4 signaling in human colorectal cancer cells increases beta 1 integrin-mediated cell adhesion and liver metastasis. Cancer Res 71(5):1989-1998. https://doi.org/10.1158/0008-5472.CAN-10-2833

18. Ikebe M, Kitaura Y, Nakamura M, Tanaka H, Yamasaki A, Nagai S et al (2009) Lipopolysaccharide (LPS) increases the invasive ability of pancreatic cancer cells through the TLR4/MyD88 signaling pathway. J Surg Oncol 100(8):725-731. https://doi.org/10.1002/jso.21392

19. Katoh H, Yamashita K, Wang G, Sato T, Nakamura T, Watanabe M (2011) Anastomotic leakage contributes to the risk for systemic recurrence in stage II colorectal cancer. J Gastrointest Surg 15(1):120-129. https://doi.org/10.1007/s11605-010-1379-4

20. Kogut MH, Iqbal M, He H, Philbin V, Kaiser P, Smith A (2005) Expression and function of Toll-like receptors in chicken heterophils. Dev Comp Immunol 29(9):791-807. https://doi.org/10.1016/j.dci.2005.02.002

21. McArdle CS, McMillan DC, Hole DJ (2005) Impact of anastomotic leakage on long-term survival of patients undergoing curative resection for colorectal cancer. Br J Surg 92(9):1150-1154. https://doi.org/10.1002/bjs.5054

22. McDonald B, Spicer J, Giannais B, Fallavollita L, Brodt P, Ferri LE (2009) Systemic inflammation increases cancer cell adhesion to hepatic sinusoids by neutrophil mediated mechanisms. Int $\mathrm{J}$ Cancer 125(6):1298-1305. https:// doi.org/10.1002/ijc.24409

23. McMillan DC, Canna K, McArdle CS (2003) The effect of deprivation and the systemic inflammatory response on outcome following curative resection for colorectal cancer. $\mathrm{Br} J$ Cancer 89(4):612-614. https://doi.org/10.1038/sj.bjc.6601156

24. Metzler KD, Fuchs TA, Nauseef WM, Reumaux D, Roesler J, Schulze I et al (2011) Myeloperoxidase is required for neutrophil extracellular trap formation: implications for innate immunity. Blood 117(3):953-959. https://doi.org/10.1182/blood-2010-06-290171

25. Miki C, Tanaka K, Inoue Y, Araki T, Ohi M, Mohri Y et al (2008) Perioperative host-tumor inflammatory interactions: a potential trigger for disease recurrence following a curative resection for colorectal cancer. Surg Today 38(7):579-584. https://doi.org/10.1007/s00595-007-3674-6 
26. Mirnezami A, Mirnezami R, Chandrakumaran K, Sasapu K, Sagar P, Finan P (2011) Increased local recurrence and reduced survival from colorectal cancer following anastomotic leak: systematic review and meta-analysis. Ann Surg 253(5):890-899.

https://doi.org/10.1097/SLA.0b013e3182128929

27. Moreira D, Zhang Q, Hossain DM, Nechaev S, Li H, Kowolik CM et al (2015) TLR9 signaling through NF-KB/RELA and STAT3 promotes tumor-propagating potential of prostate cancer cells. Oncotarget 6(19):17302-17313. https://doi.org/10.18632/oncotarget.4029

28. Mynster T, Christensen IJ, Moesgaard F, Nielsen HJ (2000) Effects of the combination of blood transfusion and postoperative infectious complications on prognosis after surgery for colorectal cancer. Danish RANX05 Colorectal Cancer Study Group. Br J Surg 87(11):1553-1562. https://doi.org/10.1046/j.1365-2168.2000.01570.x

29. Nespoli A, Gianotti L, Totis M, Bovo G, Nespoli L, Chiodini P et al (2004) Correlation between postoperative infections and long-term survival after colorectal resection for cancer. Tumori 90(5):485-490.

30. Nicoud IB, Jones CM, Pierce JM, Earl TM, Matrisian LM, Chari RS et al (2007) Warm hepatic ischemia-reperfusion promotes growth of colorectal carcinoma micrometastases in mouse liver via matrix metalloproteinase-9 induction. Cancer Res 67(6):2720-2728. https://doi.org/10.1158/00085472.CAN-06-3923

31. Nowacki MP, Szymendera JJ (1983) The strongest prognostic factors in colorectal carcinoma. Surgicopathologic stage of disease and postoperative fever. Dis Colon Rectum 26(4):263-268.

32. Peroval MY, Boyd AC, Young JR, Smith AL (2013) A critical role for MAPK signalling pathways in the transcriptional regulation of toll like receptors. PLoS One 8(2):e51243.

https://doi.org/10.1371/journal.pone.0051243

33. Pilsczek FH, Salina D, Poon KK, Fahey C, Yipp BG, Sibley CD et al (2010) A novel mechanism of rapid nuclear neutrophil extracellular trap formation in response to Staphylococcus aureus. J Immunol 185(12):7413-7425. https://doi.org/10.4049/jimmunol.1000675

34. Richards CH, Platt JJ, Anderson JH, McKee RF, Horgan PG, McMillan DC (2011) The impact of perioperative risk, tumor pathology and surgical complications on disease recurrence following potentially curative resection of colorectal cancer. Ann Surg 254(1):83-89. https://doi.org/10.1097/SLA.0b013e31821fd469

35. Sagiv JY, Michaeli J, Assi S, Mishalian I, Kisos H, Levy L et al (2015) Phenotypic diversity and plasticity in circulating neutrophil subpopulations in cancer. Cell Rep 10(4):562-573. https://doi.org/10.1016/j.celrep.2014.12.039

36. Sangaletti S, Tripodo C, Vitali C, Portararo P, Guarnotta C, Casalini P et al (2014) Defective stromal remodeling and neutrophil extracellular traps in lymphoid tissues favor the transition from autoimmunity to lymphoma. Cancer Discov 4(1):110-129. https://doi.org/10.1158/2159-8290.CD-130276 
37. Serra-Aracil X, García-Domingo MI, Parés D, Espin-Basany E, Biondo S, Guirao X et al (2011) Surgical site infection in elective operations for colorectal cancer after the application of preventive measures. Arch Surg 146(5):606-612. https://doi.org/10.1001/archsurg.2011.90

38. So EY, Ouchi T (2010) The application of Toll like receptors for cancer therapy. Int J Biol Sci 6(7):675681.

39. Urban CF, Ermert D, Schmid M, Abu-Abed U, Goosmann C, Nacken W et al (2009) Neutrophil extracellular traps contain calprotectin, a cytosolic protein complex involved in host defense against Candida albicans. PLoS Pathog 5(10):e1000639. https://doi.org/10.1371/journal.ppat.1000639

40. van den Tol PM, van Rossen EE, van Eijck CH, Bonthuis F, Marquet RL, Jeekel H (1998) Reduction of peritoneal trauma by using nonsurgical gauze leads to less implantation metastasis of spilled tumor cells. Ann Surg 227(2):242-248. https://doi.org/10.1097/00000658-199802000-00014

41. Walker KG, Bell SW, Rickard MJ, Mehanna D, Dent OF, Chapuis PH et al (2004) Anastomotic leakage is predictive of diminished survival after potentially curative resection for colorectal cancer. Ann Surg 240(2):255-259. https://doi.org/10.1097/01.sla.0000133186.81222.08

42. Weitz J, Koch M, Kienle P, Schrödel A, Willeke F, Benner A et al (2000) Detection of hematogenic tumor cell dissemination in patients undergoing resection of liver metastases of colorectal cancer. Ann Surg 232(1):66-72. https://doi.org/10.1097/00000658-200007000-00010

43. Yang HT, Wang Y, Zhao X, Demissie E, Papoutsopoulou S, Mambole A et al (2011) NF-kB1 inhibits TLR-induced IFN- $\beta$ production in macrophages through TPL-2-dependent ERK activation. J Immunol 186(4):1989-1996. https://doi.org/10.4049/jimmunol.1001003

44. Yoo DG, Floyd M, Winn M, Moskowitz SM, Rada B (2014) NET formation induced by Pseudomonas aeruginosa cystic fibrosis isolates measured as release of myeloperoxidase-DNA and neutrophil elastase-DNA complexes. Immunol Lett 160(2):186-194. https://doi.org/10.1016/j.imlet.2014.03.003

45. Zannetti C, Parroche P, Panaye M, Roblot G, Gruffat H, Manet E et al (2014) TLR9 transcriptional regulation in response to double-stranded DNA viruses. J Immunol 193(7):3398-3408. https://doi.org/10.4049/jimmunol.1400249

\section{Tables}


Table 1

Clinical characteristics of patients and operative results

\begin{tabular}{|c|c|c|c|}
\hline Parameter & $\begin{array}{l}\text { Infection group } \\
n=40\end{array}$ & $\begin{array}{l}\text { Control group } \\
n=40\end{array}$ & $p$ value \\
\hline Age (year) & $75.3 \pm 10.5$ & $74.2 \pm 9.8$ & \\
\hline Gender (male) & $33(82.5 \%)$ & $34(85 \%)$ & \\
\hline \multicolumn{4}{|l|}{ ASA status } \\
\hline 1 & $3(7.5 \%)$ & $4(10 \%)$ & \multirow[t]{4}{*}{0.2381} \\
\hline 2 & $9(22.5 \%)$ & $10(25 \%)$ & \\
\hline 3 & $24(60 \%)$ & $22(55 \%)$ & \\
\hline 4 & $4(10 \%)$ & $4(10 \%)$ & \\
\hline CEA (mg/dl) & $7.2 \pm 2.1$ & $4.7 \pm 2.3$ & $2.6 \times 10^{-5}$ \\
\hline Operative time (min) & $195.4 \pm 52.2$ & $183.7 \pm 51.3$ & 0.169 \\
\hline Laparoscopy & $5(12.5 \%)$ & $3(7.5 \%)$ & 1 \\
\hline Blood transfusion & $5(12.5 \%)$ & $4(10 \%)$ & 1 \\
\hline Reoperation & $23(57.5 \%)$ & 0 & $9.314 \times 10^{-8}$ \\
\hline Hospital stay (days) & $32.3 \pm 15.2$ & $6.5 \pm 4.2$ & $1.096 \times 10^{-13}$ \\
\hline
\end{tabular}


Table 2

Relationships between clinical variables and recurrence

\begin{tabular}{|lll|}
\hline Variable & HR (Cl 95\%) & P value \\
\hline Gender & & \\
\hline Female (reference) & 1.00 & \\
\hline Male & $1.234(0.534-3.531)$ & 7.24 \\
\hline Age (per year) & $0.967(0.914-1.241)$ & 0.321 \\
\hline CEA (per mcg/L) & $1.121(0.932-1.243)$ & 0.658 \\
\hline Lymph node involvement (yes vs. no) & $4.245(1.587-18.462)$ & 0.003 \\
\hline Tumor stage & $1.21(0.735-2.453)$ & 0.074 \\
\hline Lymphatic invasion (yes vs. no) & $0.892(0.683-2.103)$ & 0.068 \\
\hline Vascular invasion (yes vs. no) & $2.312(1.325-5.422)$ & 0.031 \\
\hline Perineural invasion (yes vs. no) & $2.562(1.103-4.235)$ & 0.023 \\
\hline Uncomplicated course (reference) & 1.00 & \\
\hline Intra-abdominal infection & $3.254(1.958-10.174)$ & 0.001 \\
\hline HR: hazard ratio; Cl: confidence interval & & \\
\hline
\end{tabular}

Figures 
A

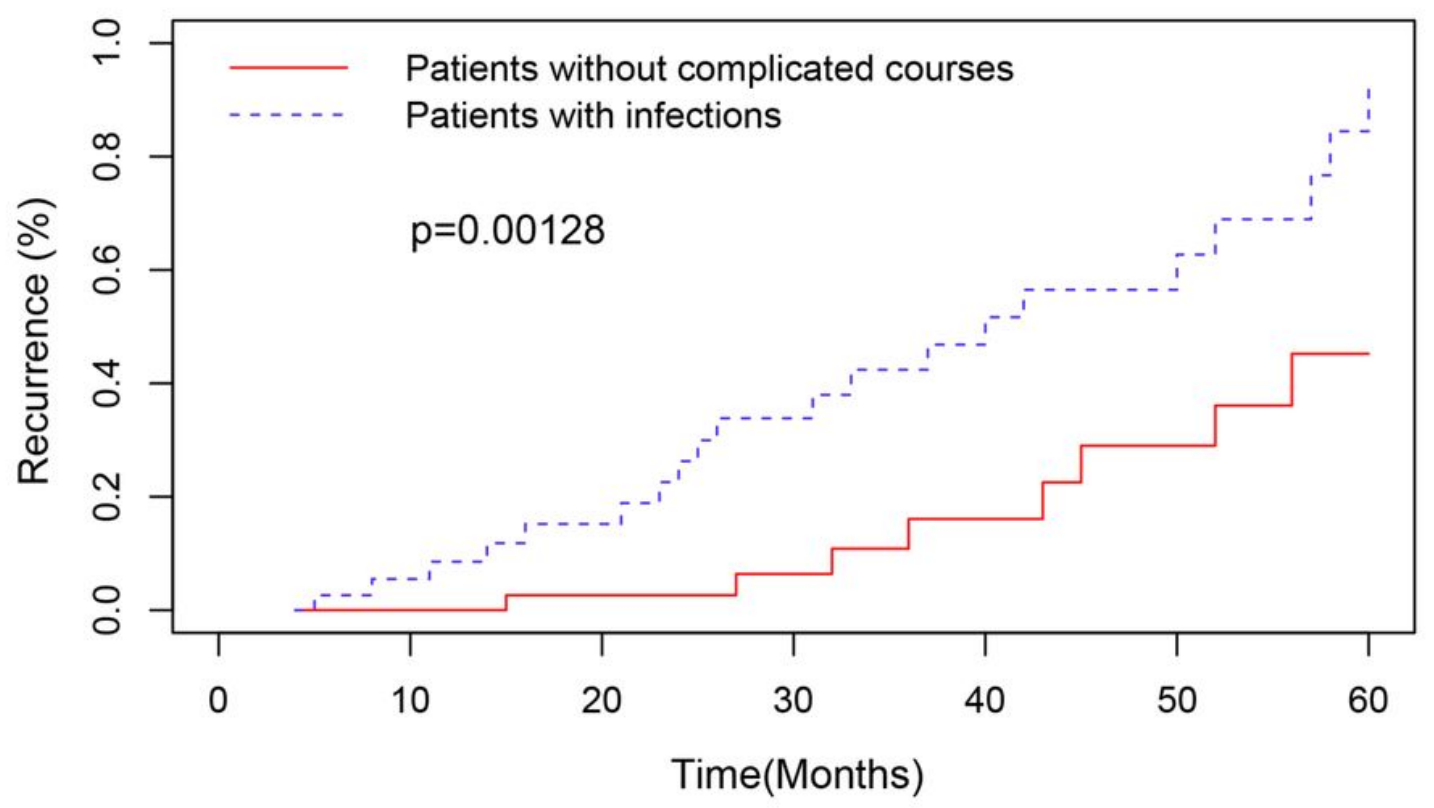

B

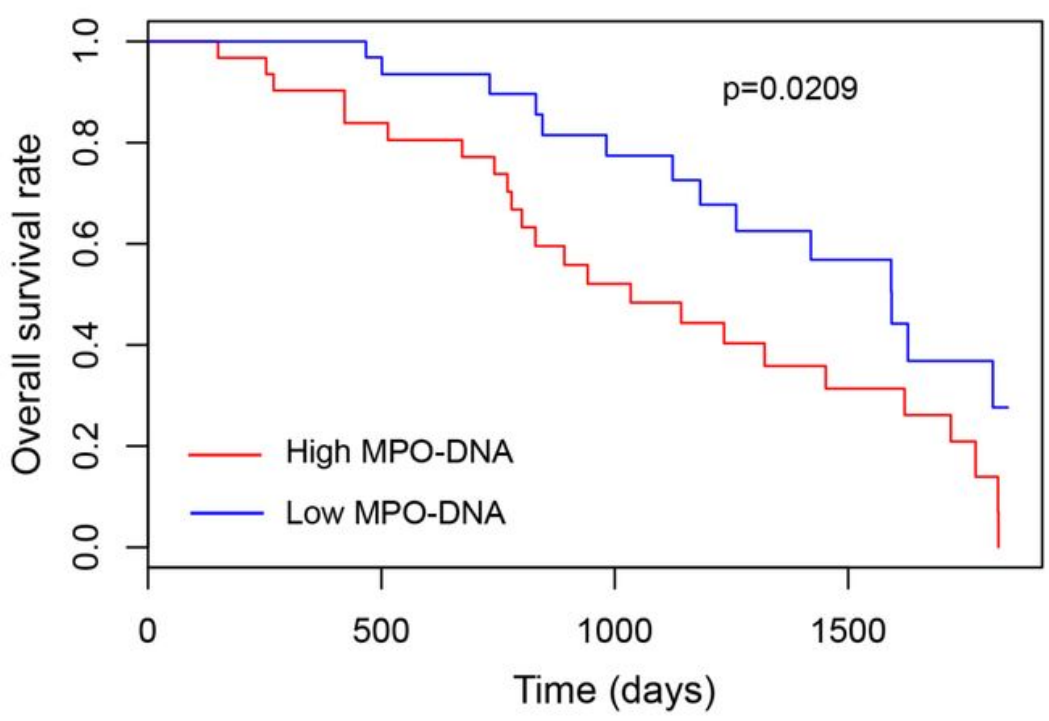

C

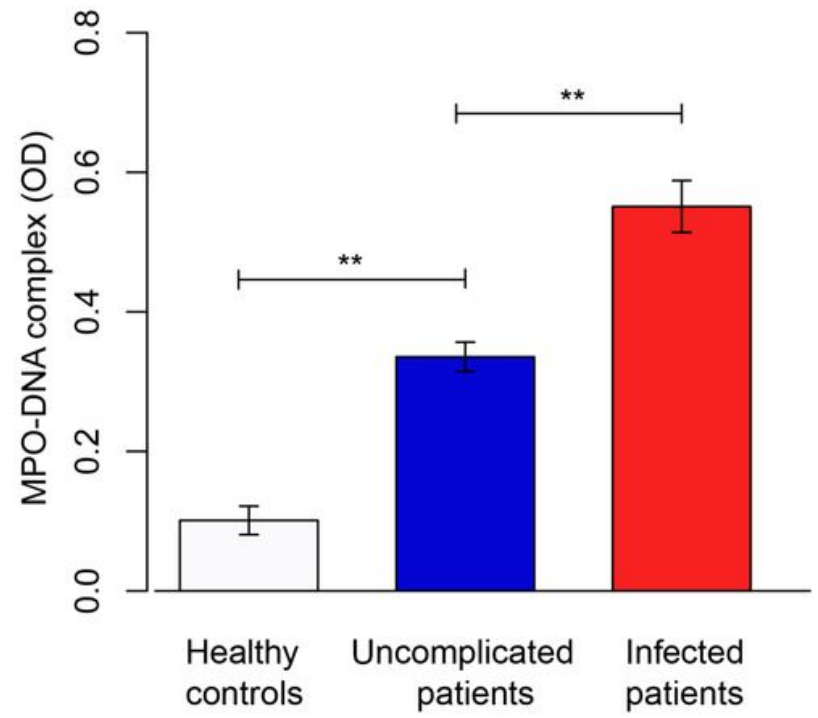

Figure 1

(A) Kaplan-Meier analysis of overall recurrence in patients with or without complicated inflammation after curative resection for colorectal cancer. (B) Levels of the MPO-DNA complex in the sera of healthy controls and recruited patients. Sera from healthy controls $(n=40)$, patients without infections $(n=40)$, and patients with infections $(n=40)$ were subjected to ELISA. ${ }^{*} p<0.01$. (C) Kaplan-Meier analysis of the overall survival rate in patients with high MPO-DNA levels and low MPO-DNA levels. Statistical significance was determined using the Mantel-Cox method. 
A

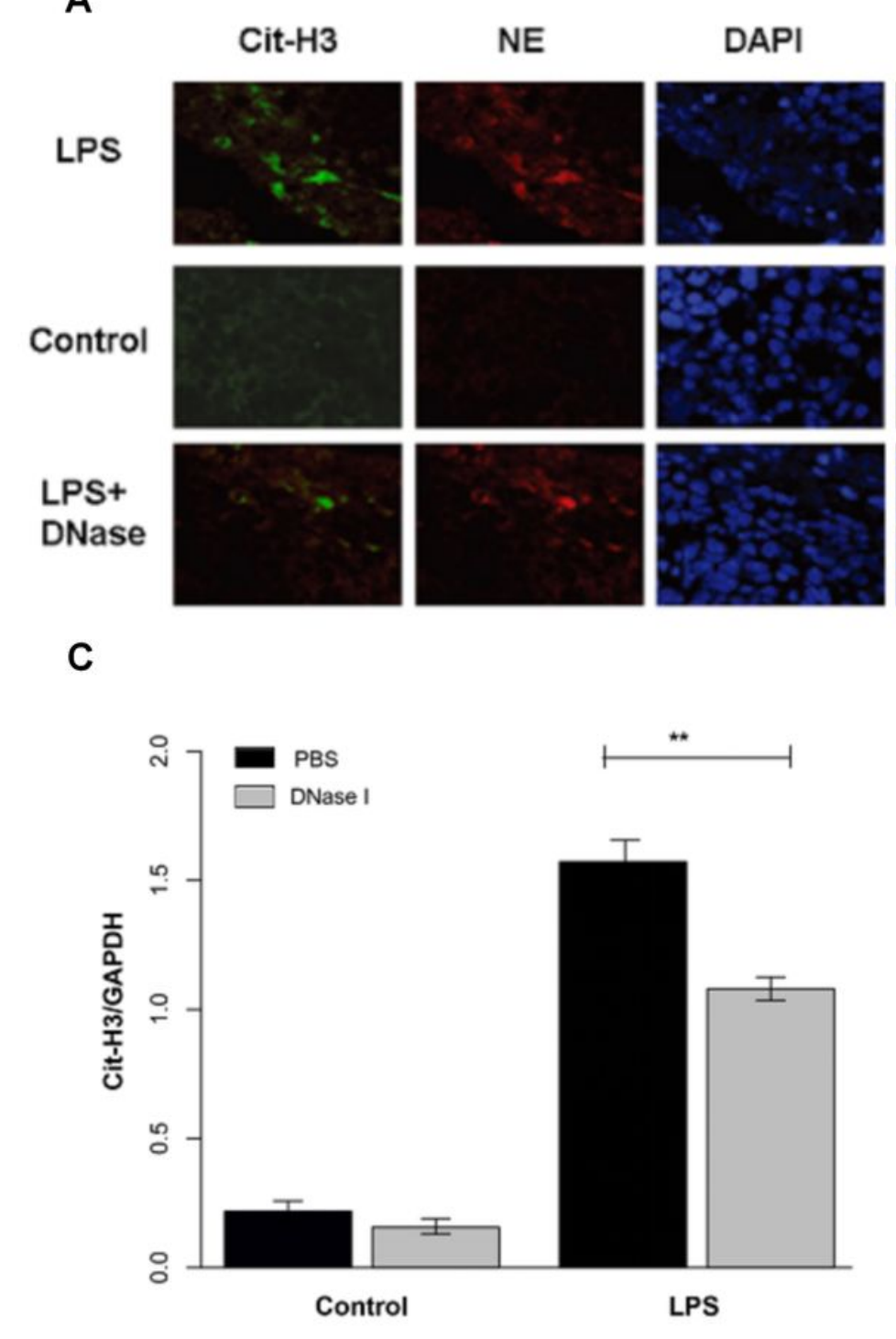

B

\section{Merge}

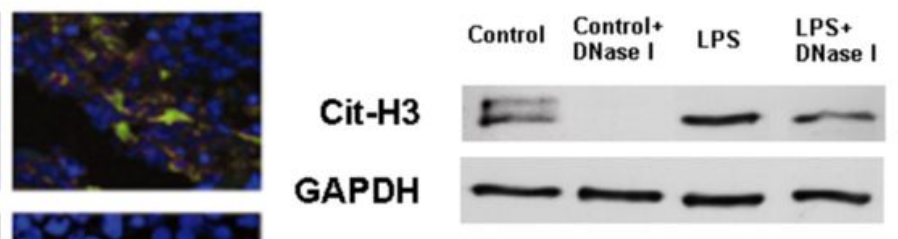

D

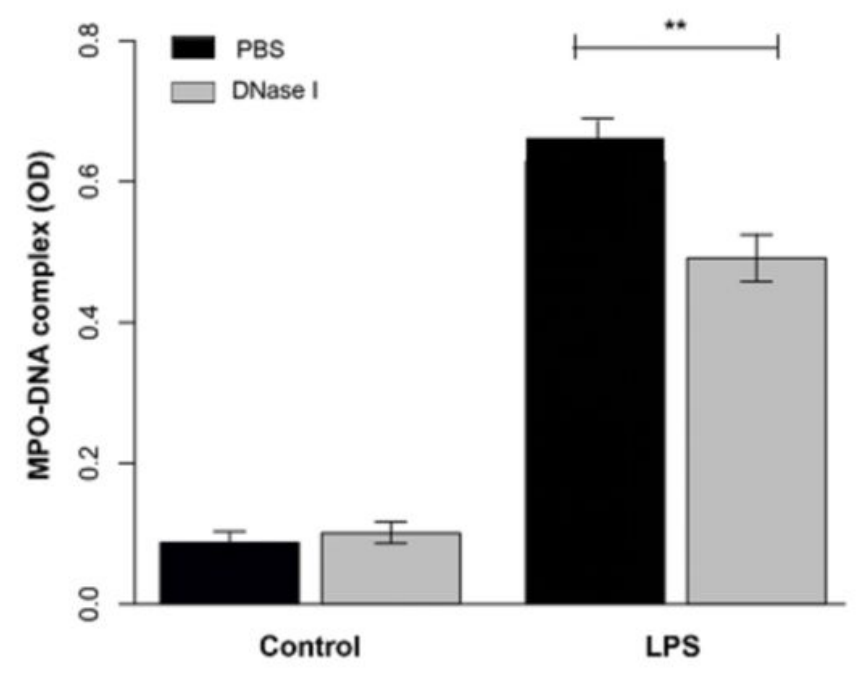

Figure 2

(A) LPS-induced formation of NETs in a mouse model of colorectal cancer. Representative immunofluorescence images obtained through confocal microscopy of colon sections from mice treated with an intraperitoneal injection of LPS. The green dye represents $\mathrm{Cit}-\mathrm{H} 3$, the red dye represents neutrophil elastase (NE), and the blue dye represents DNA. Colocalization of NE and Cit-H3 indicates the formation of NETs. (B) Western blotting for Cit-H3 in colon sections of LPS-treated mice. (C) Quantification of the level of Cit-H3 protein in colon sections of LPS-treated mice. ${ }^{\star *} p>0.01$. (D) Levels of the MPO-DNA complex in the sera of mice treated with LPS (and DNase I). 

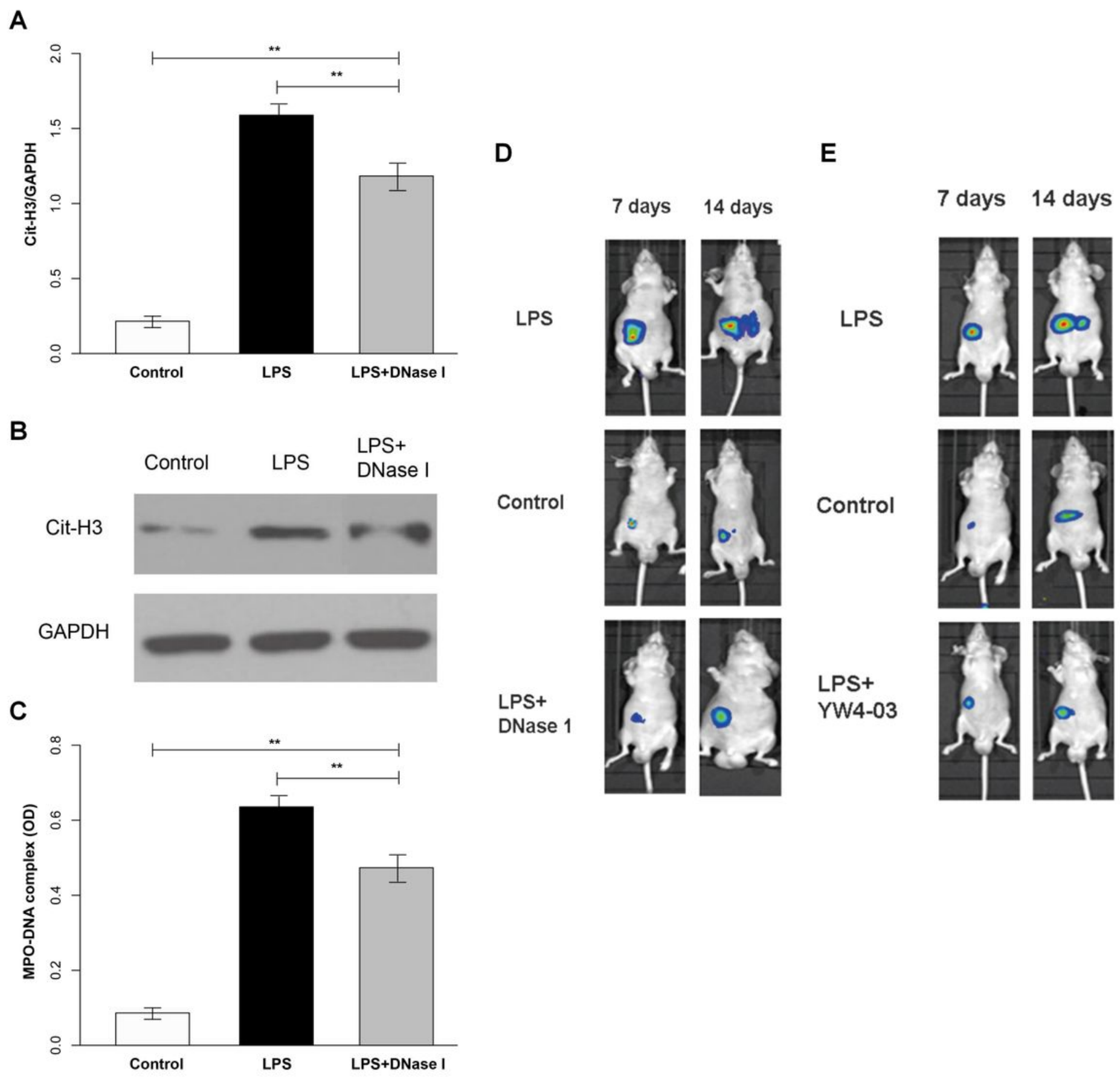

\section{Figure 3}

(A) Levels of Cit-H3 in liver tissue obtained from the hepatic metastasis mouse model with and without intraperitoneal injection of LPS (and DNase I). ${ }^{* \star} p<0.01$. (B) Western blotting for Cit-H3 in liver tissue obtained from the hepatic metastasis mouse model with and without intraperitoneal injection of LPS (and DNase I). (C) Levels of circulating MPO-DNA complex in the hepatic metastasis mouse model with and without intraperitoneal injection of LPS (and DNase I). (D) Bioluminescence imaging of metastasis of luciferase-labeled HCT116 cells in the hepatic mice model with and without LPS treatment (and DNase 
I). (E) Bioluminescence imaging of metastasis of luciferase-labeled HCT116 cells in the hepatic mice model with and without LPS treatment (and YW4-03).
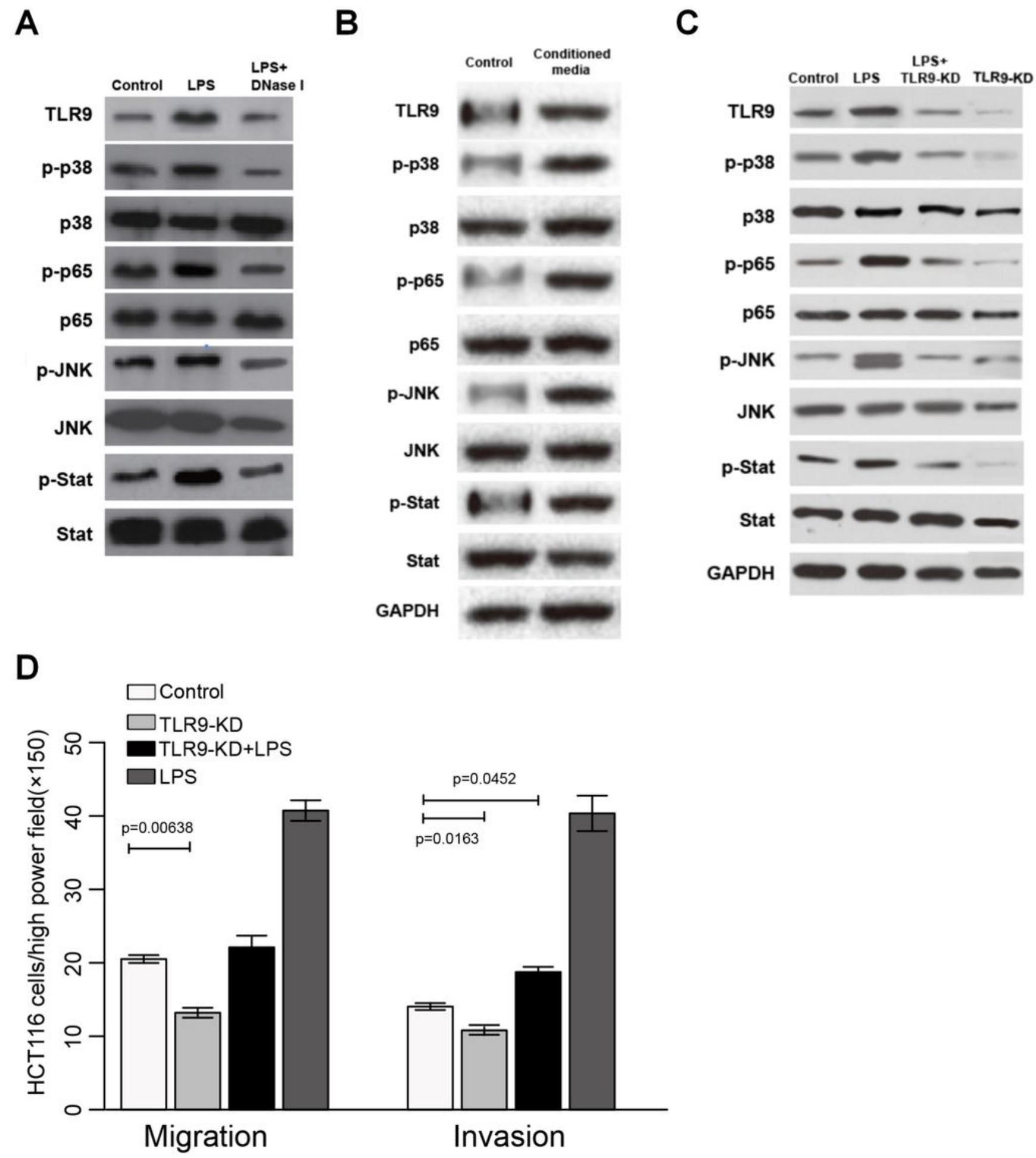

Figure 4

(A) Colon sections of mice: TLR9 and downstream proteins involved in the MAPK pathway were activated by treatment with LPS. (B) HCT116 cells cultured in media derived from LPS-induced neutrophils (conditioned): TLR9 and downstream proteins involved in the MAPK pathway. (C) Knockdown of TLR9 
results in reduced activation of proteins involved in the MAPK pathway. (D) Knockdown of TLR9 mitigates the migration and invasion capacity of HCT116 cells. 